A. Pramesh Rao, G. Swarup and Gopal-Krishna, eds.

\title{
Supernova Remnant G11.2-0.3 and the ISM
}

\author{
A.C. Seth \\ Harvard-Smithsonian Center for Astrophysics, 60 Garden St., \\ Cambridge, MA 02138, USA
}

\begin{abstract}
We present 610 and $235 \mathrm{MHz}$ GMRT observations of the supernova remnant (SNR) G11.2-0.3 which shows a very large roation measure gradient $\left(\Delta \mathrm{RM} \sim 1600 \mathrm{rad} \mathrm{m}^{-2}\right)$ across it. The GMRT observations are the first high-resolution observations of this object at low frequencies. There are differences between the structure observed at 610 and at 235 $\mathrm{MHz}$ only some of which can be explained by free-free absorption.
\end{abstract}

\section{Introduction}

The Supernova Remnant G11.2-0.3 is a bright, circular, young remnant located at $\alpha(\mathrm{B} 1950)=18^{h} 08^{m} 30^{s}$ and $\delta(\mathrm{B} 1950)=-19^{\circ} 26^{\prime}$. It has been well studied at both radio and $\mathrm{x}$-ray wavelengths. Its most notable features are a proposed (and disputed) connection with historical Supernova AD 386 and the recent discovery of an x-ray pulsar in the centre of the remnant.

A Rotation Measure map for G11.2-0.3 was derived using simultaneous 4535 and $4965 \mathrm{MHz}$ data from the Very Large Array (VLA) in C \& D arrays taken by Salter, C., Patnaik, A., Hunt, G., Shaver P. and von Gorkom, J. The rotation measure map (not shown here) clearly shows a gradient running from $\sim-300 \mathrm{radm}^{-2}$ in the southwest to $\sim-2000 \mathrm{radm}^{-2}$ in the north. Using depolarization information about this remnant we have ascertained that this gradient is external to the remnant. The gradient is therefore caused by a dramatic change in the foreground magneto-ionic medium. High resolution, low-frequency observations could allow us to disentangle the structure of the ionic medium from the magnetic field in the foreground ISM.

\section{GMRT Observations}

Observations were made with the GMRT at $610 \mathrm{MHz}$ and $235 \mathrm{MHz}$ on $17 \mathrm{Aug}$ 1999 and 8 Nov 1999. Each observation was for about 6 hours and had 21 antennas. The angular resolution was about 6 " at $610 \mathrm{MHz}$ and 17 " at 235 $\mathrm{MHz}$ and the RMS noise on the map was $0.8 \mathrm{mJy}$ per beam and $4 \mathrm{mJy}$ per beam respectively. The $610 \mathrm{MHz}$ map with 6 " resolution (Fig. 1a) agrees well with observations at higher frequencies including fine structure such as the 'ejecta' seen to the southwest (Green, 1998, MNRAS, 231, 735). However, the protrusions to the north and south are both more prominent than at $1.4 \mathrm{GHz}$.

In Figs. $1 b$ and $1 c$, we show the maps of the source at the 2 frequencies, convolved to the resolution of the $235 \mathrm{MHz}$ map. The $235 \mathrm{MHz}$ map suffered 
from astrometry problems because of which the maps are presented in relative coordinates.
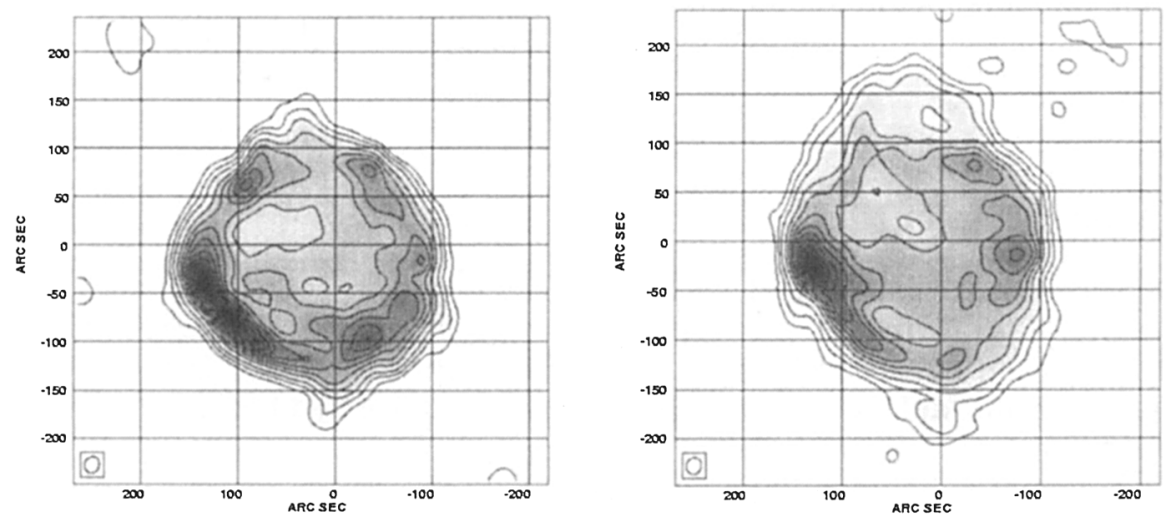

Figure 1. G11.2-0.3 (a) $610 \mathrm{MHz}, 6$ " resolution (b) $610 \mathrm{MHz}, 17^{\prime \prime}$ resolution at $(-2,2,5,10,20,30,40,50,60,70,80,90) \%$ of peak of $429.6 \mathrm{mJy} /$ beam (c) $235 \mathrm{MHz}, 17^{\prime \prime}$ resolution at $(-2,2,5,10,20,30$, $40,50,60,70,80,90) \%$ of peak of $616 \mathrm{mJy} /$ beam

Rough spectral indices for a few points in the remnant have been calculated using these maps. In many places the spectral index is consistent with the expected value of $\alpha=0.5\left(S \propto \nu^{-\alpha}\right)$. Some examples are the brightest knot in the east, the northwestern knot and the western knot seen most clearly at 235 $\mathrm{MHz}$. However, a flat spectrum $(\alpha \sim 0.0)$ is seen in the northeast and the knot just west of south, which could be caused by absorption. Using parameters from Kassim (1989, ApJ, 345, 915), we find that an extended HII envelope causing the rotation measure gradient would cause significant spectral flattening between our two frequencies for most realistic choice of temperature and magnetic field. However, the absorption does not clearly correlate with the RM gradient.

The filaments observed to the north of the remnant at $235 \mathrm{MHz}$ require a very steep spectral index $(\alpha \sim 1.7)$. This makes their reality suspect, despite the fact that they are well above the noise. If they are real, they could be caused by jets from the X-ray pulsar - there is a symmetric, though smaller, protrusion to the south. 